\title{
Teoría y Estructura de la Publicidad
}

\section{Theory and Structure of Advertising}

Fechas | En edición: 20/10/2018 - Publicación final: 01/01/2019

\section{Dra. María-Teresa GORDILLO-RODRIGUEZ}

Universidad de Sevilla. España. teresagordillo@us.es

\section{Teoría y Estructura de la Publicidad}

Autores: Juan Carlos RODRÍGUEZ-CENTENO y Jorge David FERNÁNDEZ-GÓMEZ

Madrid: Síntesis, 2017.

259 páginas

ISBN: 978-84-9171-081-3

Ebook: 978-84-9171-627-3

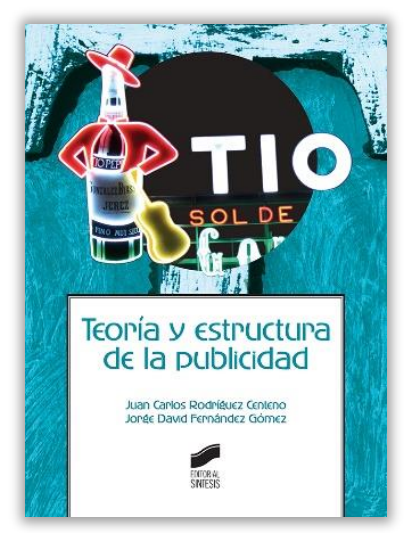

\section{Resumen}

Teoría y Estructura de la Publicidad supone un completo estudio del proceso publicitario. Desde la necesidad del anunciante hasta la difusión del mensaje, pasando por la planificación estratégica o la creación, el texto ofrece las claves de la actividad publicitaria en todas sus etapas. Se presenta la comunicación publicitaria como contexto, se explican los agentes que protagonizan el proceso comunicativo (el anunciante, la marca y la empresa de publicidad) y se desgrana el proceso en fases bien diferenciadas, siguiendo el orden lógico del proceso publicitario: la planificación de marketing, la planificación estratégica, la creatividad publicitaria, la producción y los medios de difusión.

\section{Palabras clave}

Agencia de publicidad; comunicación; estructura de la publicidad; teoría de la publicidad.

\begin{abstract}
Theory and Structure of Advertising presents a complete study of the advertising process. From the need of the advertiser to the dissemination of the message, through strategic planning or creation, this text offers the keys to advertising activity in all its stages. Advertising communication is presented as a context, as well as the agents involved in the communication process (the advertiser, the brand and the advertising company), and the process is separated into well differentiated phases, following the logical order of the advertising process: marketing planning, strategic planning, advertising creativity, production and media.
\end{abstract}

\section{Keywords}

Advertising agency; communication, structure of advertising; theory of advertising. 
El volumen Teoría y Estructura de la Publicidad se configura como un texto de naturaleza académica con voluntad pedagógica. Esta obra combina rigor en los contenidos y claridad en la exposición, por lo cual puede calificarse como material docente de alta calidad. El texto presenta una organización clara de los contenidos, que aparecen apoyados por figuras y esquemas. Además, al final de cada capítulo se incluye un glosario de términos básicos, un resumen, un caso práctico real ilustrativo y una serie de preguntas de autoevaluación; todo ello cerrado por una lista de referencias bibliográficas. Y es que sus autores, profesores de la Universidad de Sevilla, cuentan con una larga trayectoria no sólo docente sino también profesional. Con todo ello, el libro reseñado es de máxima pertinencia en el presente contexto académico, pues supone una revisión de los contenidos teóricos propios de la disciplina publicitaria a la luz de la práctica profesional actual.

En la línea de otros volúmenes publicados por el profesor Rodríguez Centeno y el profesor Fernández Gómez, este libro conjuga lo conceptual con su aplicación práctica. En particular, Teoría y Estructura de la Publicidad supone un completo estudio del proceso publicitario. Desde la necesidad del anunciante hasta la difusión del mensaje, pasando por la planificación estratégica o la fase de creación, el texto ofrece las claves de la actividad publicitaria en todas sus etapas. Dividido en nueve capítulos, el presente volumen desgrana el proceso en fases bien diferenciadas. El primer capítulo se presenta como introductorio. Titulado "La comunicación publicitaria", ofrece conceptos básicos relacionados con la disciplina y la práctica profesional, así como una breve introducción histórica. Los tres siguientes corresponden a los agentes que protagonizan el proceso comunicativo, concretamente: "El anunciante", "La marca" y "La empresa de publicidad". Los cinco últimos corresponden a las fases mencionadas, ordenadas siguiendo el orden lógico del proceso publicitario: "La planificación de marketing", "La planificación estratégica", "Creatividad publicitaria", "Producción publicitaria" y "Los medios de difusión".

Si bien esta estructura es en apariencia de carácter aplicado, los contenidos no dejan de contar con base científica, pues se apoyan en fuentes bibliográficas de autores de renombre en las disciplinas de marketing, comunicación y publicidad, tales como David Aaker, Roland Barthes, Edward de Bono, Raúl Eguizábal, Jean-Noël Kapferer, Philip Kotler, Roser Reeves, David Ogilvy, Toni Segarra o Jackes Séguéla, entre otros. Como decíamos, este volumen tiene como principal público objetivo a los estudiantes de publicidad, comunicación o marketing (aunque también a aquellos interesados en estas disciplinas de forma trasversal), lo cual justifica tanto la estructura como las fuentes: se trata de realizar un recorrido por el proceso publicitario utilizando como base teórica las referencias clave en esta área. De este modo, el lector puede tener una clara idea tanto de los cimientos de la práctica profesional como de su aplicación más inmediata.

Escrito en un estilo conciso y directo, Teoría y Estructura de la Publicidad se erige como un volumen imprescindible para los estudiantes universitarios de publicidad en particular, y de marketing y comunicación en general. Constituye una aproximación rigurosa al proceso publicitario difícil de encontrar en los títulos actuales sobre la materia. La obra, de orientación pedagógica, nace como respuesta a las nuevas necesidades docentes y conjuga a la perfección los contenidos correspondientes a la teoría y la práctica publicitaria. Considerando la experiencia de los profesores Rodríguez Centeno y Fernández Gómez, la obra se hace totalmente pertinente en el contexto actual pues explica con precisión cuáles son las claves del proceso publicitario, incluyendo perfiles profesionales, técnicas, metodologías de trabajo o tipos de empresas implicadas.

A diferencia de otras obras de corte similar, este volumen incluye un primer capítulo en el que se aclara no sólo qué es la publicidad, sino también qué no es, decisión que nos parece bastante acertada. Por ejemplo, diferenciando la publicidad de otras modalidades de comunicación persuasiva como las relaciones públicas o la propaganda. Una vez establecidos los cimientos, se pasa a definir los agentes más importantes, como son la empresa anunciante y la empresa de publicidad, y se realiza una aproximación al concepto de marca, que se nos antoja totalmente pertinente pues delimita el enfoque desde el que se va a tratar dicho concepto (sobre el que no existe consenso en la literatura académica) a lo largo del libro. El capítulo dedicado a las empresas de publicidad es de especial utilidad para los estudiantes pues los acerca al mercado real, presentando la variedad de empresas y estructuras que se pueden encontrar en la actualidad. Es también interesante que se dedique un capítulo a la planificación de marketing como marco en el que se incluye la planificación estratégica publicitaria, a la que se dedica otro capítulo independiente, diferenciando así el enfoque de marketing del enfoque publicitario. En concreto, cabe destacar que aquí se presentan de forma concisa las principales herramientas estratégicas que utilizan las agencias punteras a la hora de elaborar campañas efectivas. Con respecto al capítulo centrado en el proceso creativo, nos encontramos con el rol de la creatividad en la empresa de publicidad, así como una breve revisión de distintas técnicas creativas. El capítulo dedicado a la producción ofrece un 
completo listado de las distintas fases y personas implicadas en dicho proceso. Finalmente, una clasificación de los medios de difusión y los distintos formatos cierra el volumen reseñado.

Si bien es cierto que este texto no peca de imprecisión, es necesario puntualizar que, en el esfuerzo por incluir todos los elementos que definen la comunicación publicitaria, se ha debido sacrificar la profundidad en algunos casos. Esto es debido, con toda seguridad, a la voluntad de abarcar todo el proceso en un único volumen. Por el mismo motivo (nos referimos a la falta de espacio), otras fases como la investigación previa o el control de la eficacia de las campañas no han tenido lugar en este libro y serían interesantes para completarlo.

Se puede considerar un acierto la inclusión casos prácticos reales al final de cada capítulo para ilustrar los contenidos, pues es el modo idóneo de trasladar los contenidos de naturaleza teórica al ámbito profesional. De esta manera, se pueden tangibilizar los conceptos y comprobar cómo realmente se ven reflejados en la práctica profesional, en el día a día de las agencias de publicidad y las empresas de marketing. Nos encontramos con casos realmente interesantes como el nacimiento de la agencia VCCP Spain, el ranking de los productos más innovadores en el sector del gran consumo, la planificación estratégica realizada por VCCP Spain para una pequeña marca de colchones castellana (que llega a ganar diez premios en festivales publicitarios), o la exitosa campaña de Grey Spain para la defensa del uso del español en la publicidad. Otro aspecto destacable es la inclusión de un glosario o preguntas de autoevaluación al final de cada capítulo. A priori, estas páginas parecen restar rigor al texto, haciéndolo parecer un "cuaderno del alumno", pero nada más lejos de la realidad. La seriedad y cientificidad del texto prevalecen por encima de estos aspectos accesorios.

En definitiva, Teoría y Estructura de la Publicidad puede considerarse como un texto de gran relevancia en el ámbito de la universidad. Principalmente, por contener las claves que caracterizan al proceso publicitario completo y por desgranar sus particularidades, desde los perfiles profesionales implicados hasta las metodologías de trabajo. Se trata de una obra que recopila con bastante acierto los avances científicos más relevantes en la disciplina publicitaria en el último siglo, para traducirlos a su aplicación práctica más inmediata. Una actualización necesaria a todas luces, pues reconcilia las figuras del publicitario de agencia y el publicitario académico. 
